\title{
KNEE JOINT TUBERCULOSIS PRESENTING AS IMMUNE THROMBOCYTOPENIA
}

\author{
MOHAMMAD ABDULLAH AZ ZUBAYER KHAN ${ }^{1}$, FAZLE RABBI MOHAMMED ${ }^{1}$, MD. ZIAUSSHAMS ${ }^{2}$, \\ SHAYMAL SARKER ${ }^{3}$, MD. BILLAL ALAM ${ }^{4}$
}

\section{Introduction:}

Tuberculosis (TB) is a major public health problem of increasing proportions in Bangladesh for last several decades. A total of 350,641 cases were reported in Bangladesh in 2006 representing an incidence of 225 per 100,000 population for that year. ${ }^{1}$ Many hematological derangements such as anaemia, leukocytosis or pancytopenia can occur in association with TB. ${ }^{2}$ However isolated thrombocytopenia is rare and most commonly occur via a non immune mechanism in the setting of pancytopenia that develop secondary to granulomatous infiltration of the bone marrow. ${ }^{3}$ However immune thrombocytopenic purpura (ITP) in a patient with TB is exceedingly a rare event. ${ }^{4}$ Here we report a case of ITP associated with TB of knee joint in whom thrombocytopenia and TB were successfully treated. A casual association between joint TB and ITP is extraordinarily rare and to our knowledge this is the first such case documentation in Bangladesh, although a few case reports citing association of TB and ITP are found in literature.

\section{Case Report:}

A 21 years old previously healthy male patient of low socioeconomic condition was admitted in a medicine unit of Dhaka Medical College Hospital with new onset of acute severe hemoptysis, malaena, and haematuria for two days with purpuric rash all over the body mainly in trunk for 7 days. He had left knee joint swelling for last 6 months which was painless without any overlying skin change and rigidity. $\mathrm{He}$ also complained of evening rise of temperature for last 2 months and un-quantified involuntary weight loss for the same duration. He did not give any significant drug history. Physical examination revealed the patient was acutely ill, temperature $101^{\circ} \mathrm{F}$ orally, pulse 100 beats/minute, regular, blood pressure 90/ $60 \mathrm{~mm}$ of $\mathrm{Hg}$. There was extensive petechia and purpura mainly in the trunk. There was left knee joint swelling with raised local temperature and grade II tenderness, patellar tap was positive with mild movement restriction and quadricep and calf muscle wasting. There was no organomegaly or peripheral lymphadenopathy. All other system examination revealed normal. The initial complete blood count (CBC) showed haemoglobin $7.9 \mathrm{gm} / \mathrm{dl}$, total WBC count $8.6 \times 10^{9} / \mathrm{L}$ with $85.9 \%$ neutrophils, $11 \%$ lymphocytes, $3 \%$ monocytes., Platelet count was $10 \times 10 /$ L. The peripheral smear showed marked paucity of platelets with no evidence of haemolysis. Bone marrow aspiration showed hypercellular marrow with increased $\mathrm{M} / \mathrm{E}$ ratio and normal maturation. Megakaryocytes were increased with normal morphology. No granuloma or hemophagocyte was detected. (Fig.-1)

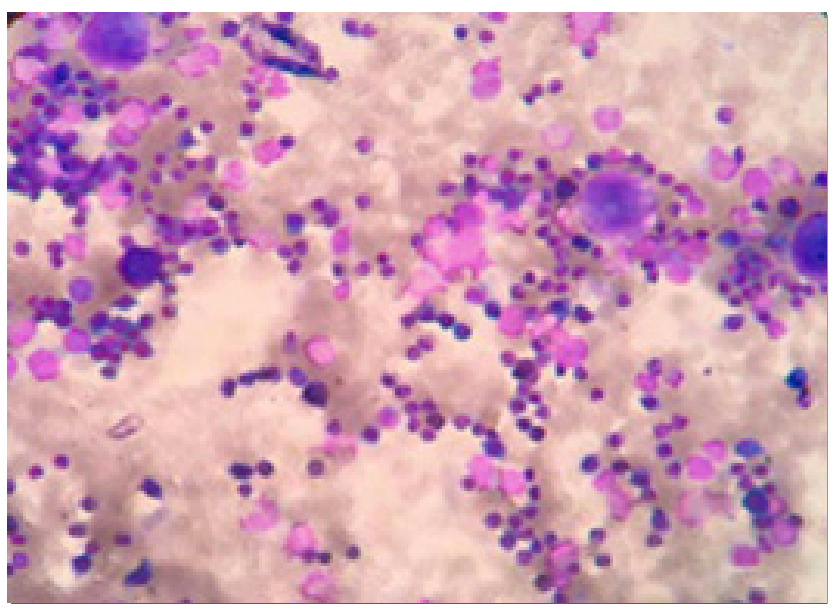

Fig.-1: Bone marrow aspirate showing plenty of megakaryocytes.

Bleeding time, clotting time, activated partial thromboplastin time, anti nuclear antibody, rheumatoid factor were within normal range.

1. Post-graduate Trainee, Department of Medicine, Dhaka Medical College \& Hospital, Dhaka

2. Assistant Registrar, Department of Medicine, Dhaka Medical College \& Hospital, Dhaka

3. Associate Professor, Department of Medicine, Mymensingh Medical College, Mymensingh

4. Associate Professor, Department of Medicine, Dhaka Medical College, Dhaka

Correspondence : Dr. Mohammad Abdullah Az Zubayer Khan, Department of Medicine, Dhaka Medical College \& Hospital, Dhaka 
Erythrocyte sedimentation rate was $40 \mathrm{~mm}$ in first hour; mantoux test was $22 \mathrm{~mm}$ induration. Serum creatinine, blood urea, blood uric acid, liver function test all were within normal limit. X-ray chest posterioranterior view revealed apparently normal parenchyma. Aspiration from left knee joint swelling showed haemorrhagic collection with plenty polymorphs, exudates and no bacteria or acid fast bacilli. Prothrombin time, fibrinogen degradation product, antibody to platelets, screening for Salmonella, Brucella, HIV, HBsAg, anti HCV and blood culture were not done due to poor financial condition of the patient.. Arthroscopy revealed multiple rice-bodies in synovial membrane; biopsy was taken and found multiple granulomas consistent with TB. (Fig.-2) Tissue culture was not done.

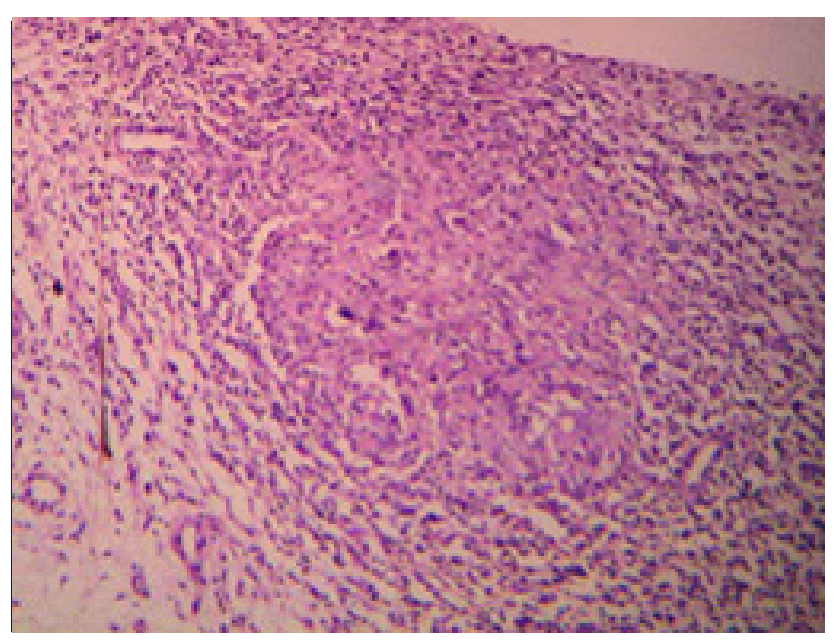

Fig.-2: Left knee joint synovial membrane biopsy showing casseous granuloma.

After admission he was treated with two units of fresh whole blood and intravenous (I/V) Dexamethasone. His condition was improving but on second week, platelet count again dropped to $10 \times 10 / \mathrm{L}$. Three units of fresh whole blood were given again and I/V Dexamethasone continued. After that platelet count was improved to $60 \times 10 / \mathrm{L}$ and biopsy was taken. According to biopsy report anti TB drugs (Rifampicin $450 \mathrm{mg}$, isoniazid $225 \mathrm{mg}$, pyrazinamide $40 \mathrm{mg}$, ethambutol $725 \mathrm{mg}$ and pyridoxine $75 \mathrm{mg}$ daily) along with oral prednisolon ( $1 \mathrm{mg} / \mathrm{kg} /$ day ) started. After ten days of treatment platelet count reached to $210 \times 10 / \mathrm{L}$. At that time patients was afebrile, bleeding manifestation subsided and wellbeing was improved. Oral steroid was tapered gradually and after 75 days of discharge platelet count became $310 \times 10 / \mathrm{L}$.

\section{Discussion:}

ITP is a relatively common immune-driven disorder that classically occurs in two forms: a childhood variety that is acute, without gender predilection, post-viral and self-limited, and the adult form that tends to be chronic, female-predominant, and most frequent between the $2^{\text {nd }}$ and 4 th decades. ${ }^{5}$ In approximately $50 \%$ of cases of ITP no underlying etiology is discovered. Many disorders may lead to secondary ITP such as systemic lupus erythematosus, lymphoproliferative disorders, especially chronic lymphocytic leukemia and hodgkin's lymphoma, and medications but tuberculosis is very rare.

The two principal diagnostic criteria for ITP are thrombocytopenia in the context of an otherwise normal blood count and a normal peripheral smear and the exclusion of conditions capable of inducing thrombocytopenia. ${ }^{6}$ The case that we report could be confused by coincidental presentation of adult idiopathic thrombocytopenic purpura and tuberculosis ; by drug-induced thrombocytopenia, thrombotic thrombocytopenic purpura (TTP), hemolytic uremic syndrome (HUS), hemophagocytic syndrome or disseminated intravascular coagulation (DIC) associated with TB. But adult ITP typically follows an insidious course with long-lasting histories of purpura (thrombocytopenia for $>6$ months) and spontaneous remission is uncommon and is likely to be incomplete. ${ }^{7-9}$ In our case, we excluded the adult ITP not only on the basis of standard criteria, but with response to steroids since thrombocytopenia did not improve up to satisfactory extent rather promptly worsen after a short rise of platelet count which might be more likely due to fresh whole blood transfusion but effect of IV dexametahsone. Also we excluded other causes of thrombocytopenia such as hemophagocytic syndrome, TTP, combined autoimmune cytopenias, hepatospleenomegaly with history, clinical and laboratoryfindings, and examination of bone marrow aspiration and biopsy that were described in case presentation. Thus the temporal association of the joint TB with bleeding manifestations, and the prompt recovery of platelet count after starting anti TB along with steroid is further evidence to suggest tuberculosis was the most likely etiology for thrombocytopenia. An immune basis for tuberculosis induced ITP was supported by the presence of either platelet antigen specific antibodies or platelet surface membrane IgG. ${ }^{10,11} \mathrm{It}$ has been postulated that the anti platelet antibodies 
generated in some cases of tuberculosis induced ITP are secreted by lymphocytes. ${ }^{12}$ In our case, antiplatelet antibody could not be detected due to in availability. However, as highlighted in the American Society for Hematology's 1996 and British Hematology of Society's 2003 guidelines for the diagnosis and management of ITP, the absence of anti-platelet antibodies in no way invalidates the diagnosis of ITP. ${ }^{13}$ In fact, anti-platelet antibodies are labeled as an "unnecessary" test for the routine evaluation to ITP. As we already mentioned ITP is a rare manifestation of tuberculosis seen in $1 \%$ of cases of adult tuberculosis in one series. ${ }^{14}$ The other reported cases of immune thrombocytopenia are case reports in association with adult pulmonary, mediastinal, disseminated ,tubercular lymphadenitis but ITP associated with joint TB have not yet reported to our best knowledge. ${ }^{6,10,15,16}$ On the basis of clinical \& histological findings that is demonstration of granuloma in synovial tissue with conventional clinical findings like evening rise of temperature, weight loss and exclusion of other causes of thrombocytopenia, the case was diagnosed as ITP due to joint TB. The patient is still under our follow up without relapsing of thrombocytopenia.

It is needless to say that TB is a new rising disease after being controlled successfully for several decades. It shows diverse presentations including different haematological abnormalities. As many questions regarding the pathophysiology, immunological factors $\&$ treatment of TB related ITP are not clear and as the incidence of this world wide disease continues to increase, further studies are required.

\section{References:}

1. WHO report 2008. Surveillance and Epidemiology, 2006. Global Tuberculosis Control. Country Profile Bangladesh. Available at: http://www.who.int/ globalatlas/ predefinedreports/tb. Accessed December 20, 2008

2. Glasser RM, Walker RI, Herion JC. The significance of hematologic abnormalities in patients with tuberculosis. Arch Intern Med 1970;125:691-695.

3. Tsuro K, Kojima H, Mitoro A, et al. Immune Thrombocytopenic Purpura Associated with Pulmonary Tuberculosis. Internal Medicine 2006; 45(11):739-742.

4. Hanta I, Gülsüm T, Kuleci S. Immune Thrombocytopenic purpura: Presenting as rare form of Tuberculosis.Turkish Respiratory Journal 2008; 9(1): 51-25.
5. George JN, Raskob GE. Idiopathic thrombocytopenic purpura. Diagnosisand management. Am J Med Sci 1998;316(2):87-93.

6. Ghobrial MW, Albornoz MA. Immune thrombocytopenia: A rare presenting manifestation of tuberculosis. Am J Hematol 2001; 67: 139-43.

7. Levine SP. Thrombocytopenia caused by immunologic plateletdestruction. In: Wintrobe's Clinical Hematology. Edited by: GreerJP, Foerster J, Lukens JN, Rodgers GM, Paraskevas F, Glader B. Philadelphia:Lippincott Williams \& Wilkins, 2004:1535-1554.

8. Ali R, Özkalemkas F, Özçelik T, et.al. Idiopathicthrombocytopenic purpura in pregnancy: a singleinstitutional experience with maternal and neonatal outcomes. Ann Hematol 2003; 82:348-352.

9. George JN, Rizvi MA. Thrombocytopenia. In: Williams Hematology. Edited by: Beutler E, Lichtman MA, Coller BS, Kipps TJ, Seligsohn U. New York: McGraw-Hill Co, 2001:1495-1540.

10. Boots RJ, Roberts AW, McEvoy D. Immune thrombocytopenia complicating pulmonary tuberculosis. Case report and Investigation of mechanism. Thorax 1992; 47: 396-7.

11. Jurak SS, Aster R, Sawaf H. Immune thrombocytopenia associated with tuberculosis. Clin Pediatr 1983; 22: 318-9.

12. Tsuro K, Kojima H, Mitoro A, et al. Immune Thrombocytopenic Purpura associated with Pulmonary Tuberculosis. Intern Med 2006;45: 739-42.

13. British Committee for Standards in Harmatology General Haematology Task Force. Guidelines for the investigation and management of idiopathic thrombocytopenic purpura in adults, children and in pregnancy. Br J Haematol 2003; 120: 574-596.

14. Al-Majed SA, Al-Momen AK, Al-Kassimi FA, AlZeer A, Kambal AM, Baagil H. Tuberculosis presenting as immune thrombocytopenic purpura. Acta Haematol 1995;94:135-138.

15. Talbot S, Dowling A, Dowling JP, Fuller A, Schwarz M. Mediastinal nodal tuberculosis presenting as immune thrombocytopenia. Aust NZ J Med 1998; 28: $465-466$.

16. Pavithran K, Vijayalekshmi N. Thrombocytopenic purpura with tuberculous adenitis. Indian J Med Sci 1993; 47: 239-240. 